\title{
Elimination of breast tumor-associated chondroitin sulfate promotes metastasis
}

\author{
R.D. Prinz ${ }^{4}$, C.M. Willis ${ }^{1,4}$, A. Viloria-Petit ${ }^{3}$ and M. Klüppel ${ }^{1,2,4}$ \\ ${ }^{1}$ Department of Pediatrics, Feinberg School of Medicine, \\ Northwestern University, Chicago, IL, USA \\ ${ }^{2}$ Robert H. Lurie Comprehensive Cancer Center, \\ Feinberg School of Medicine, Northwestern University, Chicago, IL, USA \\ ${ }^{3}$ Department of Biomedical Sciences, Ontario Veterinary College, \\ University of Guelph, Guelph, Canada \\ ${ }^{4}$ Human Molecular Genetics Program, \\ Children's Memorial Research Center, Chicago, IL, USA \\ Corresponding author: M. Klüppel \\ E-mail: m-kluppel@northwestern.edu
}

Genet. Mol. Res. 10 (4): 3901-3913 (2011)

Received June 20, 2011

Accepted October 18, 2011

Published December 8, 2011

DOI http://dx.doi.org/10.4238/2011.December.8.9

\begin{abstract}
Breast cancer is one of the leading causes of cancer-related deaths amongst women in the USA. The tumor microenvironment has been suggested to be an attractive therapeutic target for treatment of cancers. The glycosaminoglycan chondroitin sulfate, as part of the cellular microenvironment, consists of long linear chains of repeating disaccharide units, which are covalently attached to core proteins to form chondroitin sulfate-proteoglycans. In vitro studies have implicated chondroitin sulfate in various aspects of carcinogenesis, whereas the in vivo roles of chondroitin sulfate are less clear. Drastically elevated levels of chondroitin sulfate have been observed within the stromal compartment of many solid tumors, including human breast carcinomas, the significance of which is unknown. We examined the role of tumor-associated chondroitin sulfate in breast cancer progression. Enzymatic elimination of endogenous chondroitin sulfate by intra-tumor injections of chondroitinase $\mathrm{ABC}$ leads to the development of secondary tumors and increased lung metastases, while primary orthotopic
\end{abstract}


tumor growth was not affected. These results establish a metastasis-inhibiting effect of primary breast tumor-associated chondroitin sulfate, which may open novel carbohydrate-based therapeutic strategies to combat breast cancer.

Key words: Breast cancer; Orthotopic tumor; Metastasis; Chondroitinase $\mathrm{ABC}$; Chondroitin sulfate

\section{INTRODUCTION}

The tumor microenvironment has been suggested to be an attractive therapeutic target for treatment of cancers, as it is able to control tumor cell phenotype (Hendrix et al., 2003; Wegrowski and Maquart, 2004; Hendrix et al., 2007; Kenny et al., 2007; LaBarge et al., 2007). The glycosaminoglycan (GAG) chondroitin sulfate as part of the cellular microenvironment is comprised of long chains of alternating disaccharide units, attached to a tetrasaccharide linker region which links this GAG to serine residues of core proteins to form chondroitin sulfate-proteoglycans (CSPGs) (Habuchi, 2000; Kusche-Gullberg and Kjellen, 2003; Heinegard, 2009; Klüppel, 2010). Chondroitin sulfate has been recognized to play important roles in a number of biological processes that are related to human disease. For example, loss of cartilage integrity in osteoarthritis is associated with degradation of CSPGs (Thiele et al., 2004; Krasnokutsky et al., 2007; Hochberg, 2010; Wandel et al., 2010). We have previously shown that a mouse strain carrying a mutation in the chondroitin sulfotransferase gene $C 4 s t-1 / C h s t 11$ developed severe cartilage defects and osteoarthritis-like features, demonstrating a role of specific sulfation patterns of chondroitins in cartilage health (Klüppel et al., 2002, 2005). Several laboratories have also begun to elucidate potential roles for chondroitin sulfates and CSPGs in tumor biology. Strikingly increased levels of chondroitin sulfates and CSPGs have been observed in the microenvironment of a number of human solid tumors, including melanoma, ovarian adenocarcinomas, hepatocarcinoma, prostate tumors, colon adenocarcinoma, and breast cancer (Olsen et al., 1988; Kovalszky et al., 1990; Alini and Losa, 1991; Ricciardelli et al., 1997, 1999, 2009; Vijayagopal et al., 1998; Suwiwat et al., 2004; Pothacharoen et al., 2006; Sakko et al., 2008; Teng et al., 2008; Kalathas et al., 2009). The functional significance of this observation for cancer progression is unknown, but increased deposition of chondroitin sulfates within malignant tissues raises the possibility of a cancer therapy targeted towards tumor-associated chondroitin sulfates. Supporting the notion of a functional role of chondroitin sulfates in cancer cell behavior, a number of in vitro studies have accumulated experimental evidence suggesting that cancer cell-associated chondroitin sulfates could affect aspects of tumor cell behavior. For example, chondroitin sulfates mediate the interaction of breast cancer cells with P-, L- and E-selectin proteins on endothelial cells (Kawashima et al., 2000, 2002; Luo et al., 2001; Monzavi-Karbassi et al., 2007; Cooney et al., 2011). The activation of MMP-2 on melanoma cells, and invasive properties of these cells in vitro, can be modulated by chondroitin sulfates (Iida et al., 2007). Very recently it was shown that digestion of cell surface chondroitin sulfates on mouse Lewis Lung Carcinoma cells by treatment with the bacterial enzyme chondroitinase $\mathrm{ABC}$ interfered with organ colonization of these cells after tail vein injection into mice (Li et al., 2008). The same result was obtained by coinjection of lung cancer cells and a double-sulfated chondroitin sulfate (CS-E), suggesting that CS-E side chains on lung cancer cells might play a role in aspects of the homing of circulating lung cancer cells to sites of metastasis. Similar results were obtained when investigating the roles of CS-E in liver tumor formation by mu- 
rine osteosarcoma cells injected into the tail vein of mice (Basappa et al., 2009). These results point towards functional roles of chondroitin sulfates during tumorigenesis. However, it is not clear what roles, if any, the increased deposition of chondroitin sulfate within primary malignant tissues plays during cancer progression, since studies depleting chondroitin sulfate in tumors have, to our knowledge, not been performed. In an initial step toward a better understanding of this phenomenon, and to investigate the feasibility of a cancer therapy targeted towards tumor-associated chondroitin sulfates, we here describe the functional importance of endogenous chondroitin sulfate as a negative regulator of breast cancer metastasis in orthotopic mouse tumor models. Our results suggest the potential of novel carbohydrate-based therapeutic avenues to combat breast cancer progression.

\section{MATERIAL AND METHODS}

\section{Reagents and antibodies}

Chondroitinase ABC (protease-free) was obtained from The Associates of Cape Cod (East Falmouth, MA, USA), and aliquoted and handled as specified by the manufacturer (Seikagaku Inc, Japan). Antibodies included mouse-anti-C4S (MAB2030, Millipore, Billerica, MA, USA) for the detection of digested chondroitin sulfates, goat-anti-Vimentin (C-20, Santa Cruz Biotechnology Inc., USA), rabbit-anti-PECAM-1 (sc1506-R, Santa Cruz Biotechnology Inc., Santa Cruz, CA, USA). Appropriate Alexa-Fluor-conjugated secondary antibodies were purchased from Invitrogen, USA.

\section{Orthotopic mouse tumor models}

Mouse EMT6 epithelial mammary carcinoma cells (CRL-2755), ATCC, USA) were maintained in 2-dimensional cultures and used in orthotopic studies as previously described (Viloria-Petit et al., 2009; Viloria-Petit and Wrana, 2010 ). For orthotopic procedures, 1 x $10^{5}$ EMT6 cells were implanted orthotopically into the right $4^{\text {th }}$ inguinal mammary fat pad of $10-12$ week old syngeneic female Balb/c mice, which were anesthetized with isoflurane inhalation at 1 to $2 \%$. After the primary orthotopic breast tumor was established and had a volume of approximately 100-200 $\mathrm{mm}^{3}$, two groups of 6 animals each received bi-daily intra-tumor injections of $25 \mu \mathrm{L}$ of a chondroitinase $\mathrm{ABC}$ solution at a concentration of $10 \mathrm{mU} / 25 \mu \mathrm{L}$, or $100 \mathrm{mU} / 25 \mu \mathrm{L}$, in Tris-acetate buffer. Intra-tumor injections of Tris-acetate buffer alone without chondroitinase $\mathrm{ABC}$, and no injections at all, served as controls. Intra-tumor injections were performed at days 9, 11, 13, 15 and 17 after orthotopic implantation. Primary tumor volume was measured by caliper and calculated with a modified ellipsoidal formula (Volume $=0.5 \times \mathrm{D}_{\mathrm{S}}{ }^{2} \times \mathrm{D}_{\mathrm{L}}$ ) on a bi-daily basis. Animals were sacrificed when primary tumors reached a volume of $1700 \mathrm{~mm}^{3}$. Lungs were removed, and one half was fixed in Bouin's Fluid for counting of macroscopic lung metastases using a dissection microscope. The other half of the lung was fixed and embedded in OCT media for cryosectioning, followed by H\&E staining and quantitative analysis of micro-metastases. All animal procedures were approved by the appropriate Investigational Review Board of the Animal Care and Use Committee.

\section{Histology and immunofluorescence}

Primary tumor tissues and lungs were dissected in PBS and fixed in cold 4\% PFA/PBS overnight. Tissues were rinsed in cold PBS and cryo-protected by shaking the tissues in cold 0.5 
M sucrose/PBS for 12-24 h. Tissues were then embedded in O.C.T. compound (Tissue-Tek ${ }^{\circledR}$, USA), snap-frozen in a dry-ice/ethanol bath and subsequently stored at $-70^{\circ} \mathrm{C}$. Tissues were cryosectioned at $12 \mathrm{~mm}$ thickness, air-dried, re-fixed in 4\% PFA/PBS for 15 min, washed 3 times in PBS, and either stained with $\mathrm{H} \& \mathrm{E}$, or processed for immunofluorescence by permeabilization with $0.25 \%$ Triton X-100 in PBS for 7 min, followed by 3 PBS washes. Mouse primary antibodies were used in conjunction with a MOM mouse-on-mouse kit in accordance to manufacturer instructions, whereas other antibodies were used by blocking in 10\% FBS/PBS, followed by over-night incubation of primary antibody in blocking solution at $4^{\circ} \mathrm{C}$ in a humidifying chamber. Sections were then washed 3 times with PBS, followed by incubation with secondary fluorophore-conjugated Alexa antibodies (Invitrogen) for one hour at room temperature in a humidifying chamber. Sections were then washed in PBS twice, stained with DAPI for 2 minutes, washed twice in PBS again, and subsequently mounted in Prolong Gold Antifade Reagent (Invitrogen). Sections were photographed on Leica upright or inverted microscopes, and images were processed in Adobe Photoshop.

\section{RESULTS}

\section{Intra-tumor injections of $\mathrm{Ch} \mathrm{ABC}$ lead to occurrence of large secondary tumors}

In order to investigate the roles of endogenous chondroitin sulfates in breast cancer cell behavior in vitro, we utilized treatment with the bacterial enzyme chondroitinase $\mathrm{ABC}$ (ChABC) to eliminate chondroitin sulfate side chains of chondroitin sulfate proteoglycans (CSPG). For this, $1 \times 10^{5}$ EMT6 breast cancer cells were implanted orthotopically into the right 4th inguinal mammary fat pad of 10-12 week old syngeneic female Balb/c mice. After the primary orthotopic breast tumor was established and had a volume of approximately $100-200 \mathrm{~mm}^{3}$, we aimed to eliminate tumor-associated chondroitin sulfates by bi-daily intra-tumor injections of $\mathrm{ChABC}$ at days 9,11 , 13,15 and 17 after orthotopic implantation. Animals received injections of $10 \mathrm{mU} \mathrm{ChABC} / 25 \mu \mathrm{L}$ buffer (10 mU ChABC), $100 \mathrm{mU}$ ChABC/25 $\mu \mathrm{L}$ buffer (100 mU ChABC), $25 \mu \mathrm{L}$ buffer without ChABC (buffer), or no injections at all (no inj.). Each experimental group contained six animals. Primary tumor volume was measured by caliper and calculated on a bi-daily basis. This analysis showed no difference in primary tumor growth between experimental groups, demonstrating that elimination of tumor-associated chondroitin sulfates did not affect tumor growth (Figure 2A). However, in $50 \%$ of animals receiving $100 \mathrm{mU} \mathrm{ChABC} / 25 \mu \mathrm{L}$ ChABC, we did observe the occurrence of large ipsilateral secondary tumors (Figure 2E). Secondary tumors were not observed in animals receiving no injections (Figure 2A), buffer injections (Figure 2B), or injections of $\mathrm{ChABC}$ at $10 \mathrm{mU} / 25 \mu \mathrm{L}$ (Figure $2 \mathrm{C}$ ). Figure $2 \mathrm{~F}$ shows the time course of animals free of secondary tumors during these experiments, and illustrates that secondary tumors only developed after the onset of intra-tumor injection. These data demonstrated that intra-tumor injections of ChABC at $100 \mathrm{mU} \mathrm{ChABC} / 25 \mu \mathrm{L}$ could lead to the formation of secondary tumors, but did not affect primary breast tumor growth.

\section{Elimination of chondroitin sulfate leads to an increase in pulmonary metastases}

We next analyzed the metastatic burden on our animals. EMT6 breast cancer cells frequently metastasize to lung; thus, animals were sacrificed and half of the lungs were treated with Bouin's Fixative, which aids in the visualization of macroscopic lung metastases. Lungs 


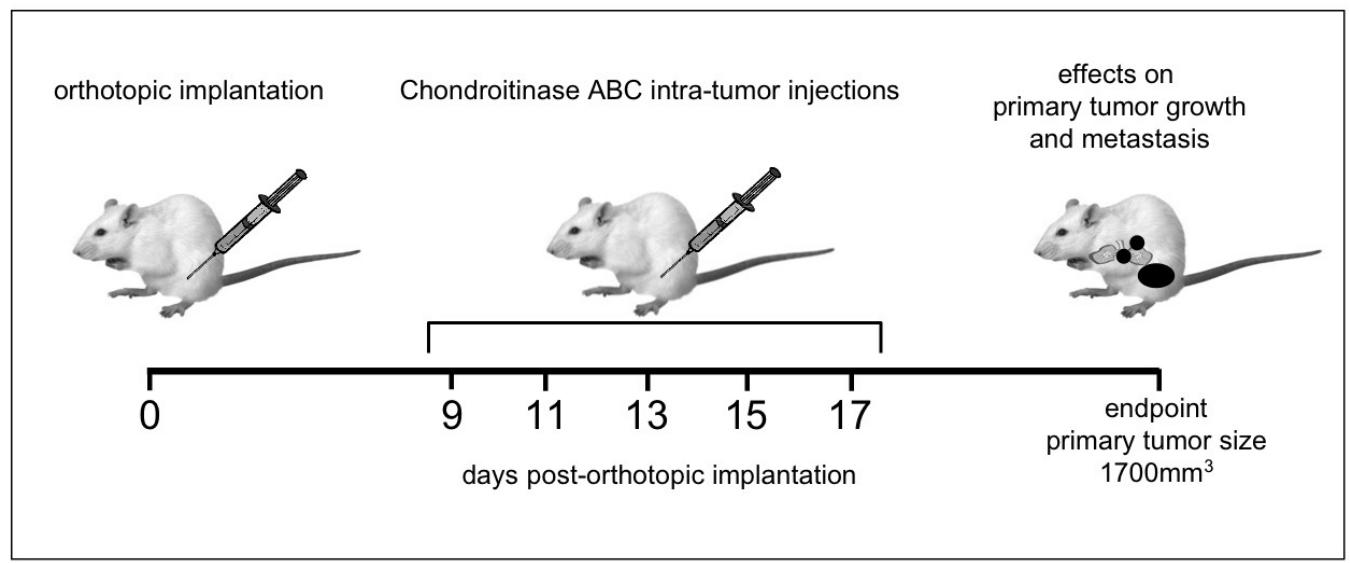

Figure 1. Experimental procedures. EMT6 cells were orthotopically implanted into the mammary gland fat pad. At days $9,11,13,15$, and 17 post-implantation, intra-tumor injections of buffer, $10 \mathrm{mU}$ ChABC or $100 \mathrm{mU}$ ChABC (all in $25 \mu \mathrm{L}$ ) were performed. Animals were sacrificed when the primary tumor reached a volume of $1700 \mathrm{~mm}^{3}$, followed by analysis of tumor growth and metastasis.
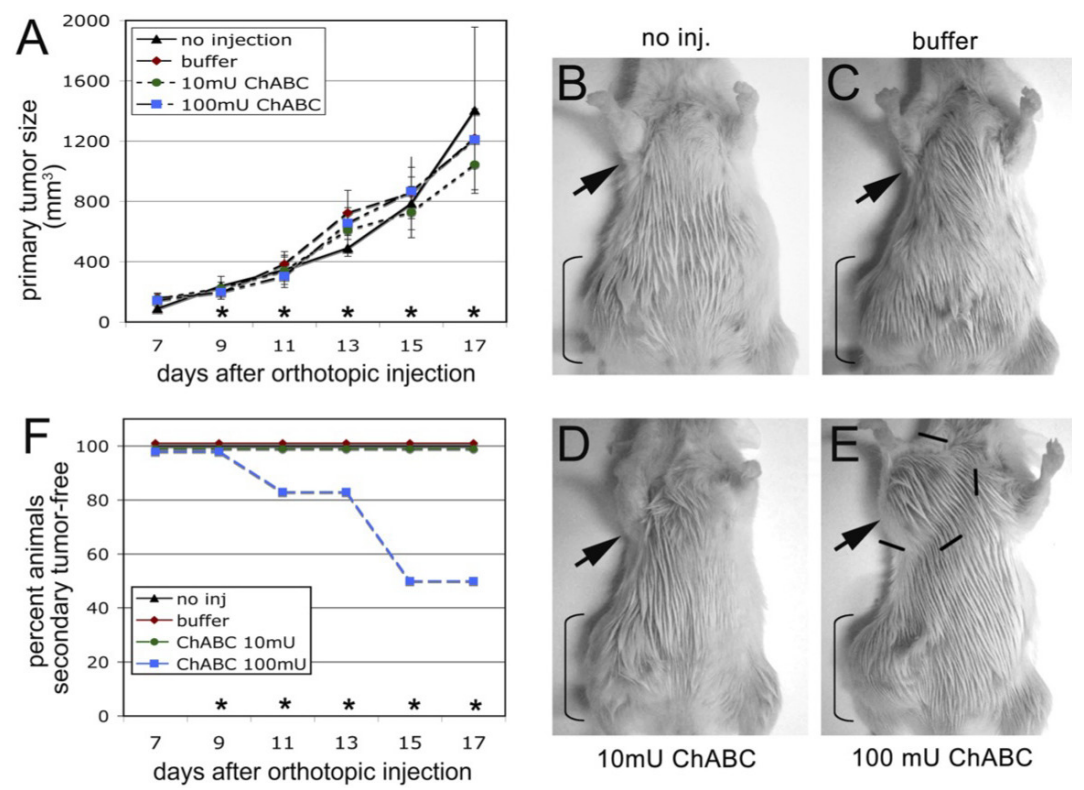

Figure 2. The effects of chondroitinase $\mathrm{ABC}$ intra-tumor injections on primary tumor growth and metastases. A. Analysis of primary tumor growth from day 7 to day 17 after orthotopic implantation. Times of intra-tumor injections are indicated (*). ChABC injections did not significantly affect primary tumor growth. B-E. Primary and secondary tumors in experimental groups. Animals receiving no injections (no inj., B), buffer injections (buffer, C) or injections of $10 \mathrm{mU}$ ChABC (D) displayed no secondary tumors (arrows), whereas injection of $100 \mathrm{mU}$ ChABC promoted the occurrence of ipsilateral large secondary tumors (arrow, E). The location of the primary breast tumor is indicated by brackets in (B-E). F. Analysis of secondary tumor occurrence from day 7 to day 17 after orthotopic implantation. Times of intra-tumor injections are indicated (*). After the onset of intra-tumor injections of ChABC at $100 \mathrm{mU}$, the development of secondary tumors was observed in $50 \%$ of animals injected. Secondary tumors did not develop in the other experimental groups. Each group consisted of six mice. 
of animals that had received no intra-tumor injections (Figure 3A), buffer injections (Figure 3B), or injections of low concentrations of ChABC (Figure 3C) displayed few small macroscopic metastasis on the lung surface. However, lungs of animals that had received intra-tumor injections of high concentrations of $\mathrm{ChABC}$ showed increased numbers of metastases, and
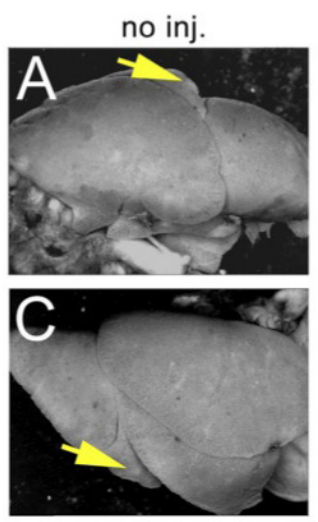

$10 \mathrm{mU}$ ChABC
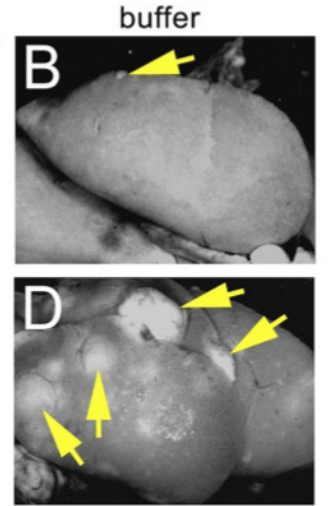

$100 \mathrm{mU}$ ChABC
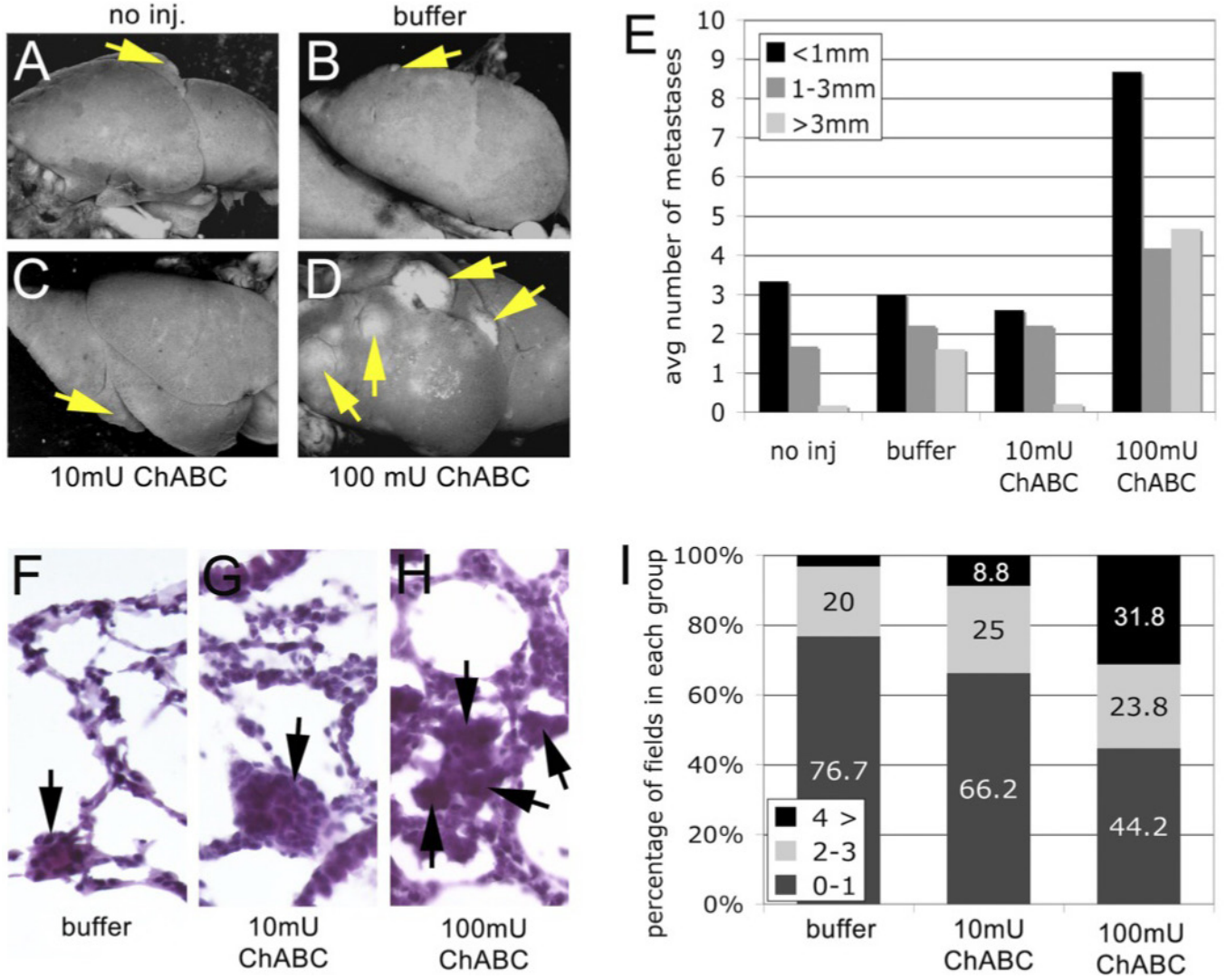

Figure 3. The effect of chondroitinase $A B C$ intra-tumor injections on lung metastases. A-D. Staining of dissected lungs with Bouin's fixative to visualize macroscopic metastases (yellow arrows). No injections (no inj., A), buffer injection (buffer, B), or injection of $10 \mathrm{mU}$ ChABC (C) lead to the development of few macroscopic metastases. D. Injection of $100 \mathrm{mU}$ ChABC promoted the occurrence of increased numbers and larger macroscopic metastases. E. Quantification of macroscopic metastases. Treatment with $100 \mathrm{mU}$ ChABC increased the average number of small $(<1 \mathrm{~mm})$, medium-sized $(1-3 \mathrm{~mm})$ and large $(>3 \mathrm{~mm})$ metastases when compared to the other experimental groups. F-H. H\&E staining of lungs to visualize micrometastases. Lungs from animals treated with buffer $(\mathbf{F})$ or $10 \mathrm{mU} \operatorname{ChABC}(\mathbf{G})$ displayed few micrometastases (arrows), whereas treatment with $100 \mathrm{mU}$ ChABC $(\mathbf{H})$ lead to increased numbers of micrometastases. Scale bar in $(\mathbf{H})=20 \mathrm{~mm}$. I. Quantification of micrometastases from H\&E stains. Number of micrometastases in microscopic fields were counted and expressed as percentage of fields with no or one (0-1), two or three (2-3), or four or more $(4>)$ metastases. In buffer-injected animals, $76.7 \%$ of fields showed no or one metastasis, whereas $20 \%$ of fields had two or three metastases, and $3.3 \%$ of fields had four or more micrometastases. Lungs of animals injected with $10 \mathrm{mU}$ ChABC had $66.2 \%$ of fields showing no or one, $25 \%$ showing two or three, and $8.8 \%$ showing four or more metastases. Lungs of animals injected with $100 \mathrm{mU}$ ChABC had $44.2 \%$ of fields showing no or one, $23.8 \%$ showing two or three, and $31.8 \%$ of fields showing four or more metastases. 
metastases were often larger than those observed in the other groups (Figure 3D). Quantification of small $(<1 \mathrm{~mm})$, medium-sized $(1-3 \mathrm{~mm})$ and large $(>3 \mathrm{~mm})$ macroscopic metastases (Figure 3E) demonstrated an almost three-fold increase in small metastases, an approximately two-fold increase in the average number of medium-sized metastases, and a three- to over 10 -fold increase in the average number of large metastases in animals that had received high concentrations of $\mathrm{ChABC}$, when compared to the other groups (Figure 3E). Next, lung tissues of animals treated with buffer, $10 \mathrm{mU} \mathrm{ChABC}$ and $100 \mathrm{mU}$ ChABC were sectioned and stained with $\mathrm{H} \& \mathrm{E}$ for identification and quantification of micrometastases (Figure $3 \mathrm{~F}-\mathrm{H}$ ). While lungs of buffer-injected animals (Figure 3F) and animals injected with 10mU ChABC (Figure 3G) showed low numbers of micrometastases, the lungs of animals injected with $100 \mathrm{mU}$ ChABC (Figure $3 \mathrm{H}$ ) appeared to display an increased number of micrometastases. To quantify these data, we analyzed the number of metastases per microscopic field (Figure 3I). In buffer-injected animals, $76.7 \%$ of fields showed no or one metastasis, whereas $20 \%$ of fields had two or three metastases. Only $3.3 \%$ of fields had four or more micrometastases. Lungs of animals injected with $10 \mathrm{mU}$ ChABC showed a slight increase in numbers of micrometastases, with $66.2 \%$ of fields showing no or one, $25 \%$ showing two or three, and $8.8 \%$ showing four or more metastases. Lungs of animals injected with $100 \mathrm{mU}$ ChABC displayed a more dramatic increase in numbers of micrometastases, with only $44.2 \%$ of fields showing no or one, $23.8 \%$ showing two or three, but $31.8 \%$ of fields showing four or more metastases. Thus, the number of microscopic fields with four or more micrometastases increased almost ten-fold in animals that received intra-tumor injections of high doses of ChABC, when compared to buffer injected controls. Taken together, these data demonstrate that treatment with ChABC increased the lung metastatic burden in this orthotopic breast cancer model.

\section{Histology of orthotopic primary tumors}

We next sectioned tumors for histological analysis. Due to the results described above, we concentrated here on a comparison of tumors from animals that had received buffer intratumor injections versus ChABC injections at $100 \mathrm{mU}$. H\&E staining of tumors revealed no major differences in general staining between tumors that were treated with buffer (Figure $4 \mathrm{~A}$ ) and tumors treated with ChABC (Figure 4B). We next wanted to confirm that ChABC injections indeed digested tumor-associated chondroitin sulfates. Immunofluorescence staining using a $\mathrm{C} 4 \mathrm{~S}$ antibody directed against proteoglycan-tetrasaccharide linker regions, which have previously been shown only to become accessible to the antibody after digestion of attached chondroitin sulfate chains, demonstrated strong staining in ChABC-treated tumors (Figure 4D), when compared to buffer-treated tumors (Figure 4C). We did, however, observe some background staining with this mouse-derived monoclonal antibody, and, maybe not unexpected, not all areas of the tumors stained positive for digested C4S, suggesting that even a multiple injection regimen could not lead to uniform distribution of $\mathrm{ChABC}$, and chondroitin sulfate digestion, throughout the tumor (data not shown). Of note, tumors that had received injections of lower doses of ChABC did not show strong C4S staining (data not shown), indicating that efficient digestion of tumor-associated chondroitin sulfate requires relatively high levels of ChABC. This is in agreement with our observations above, which demonstrated that intra-tumor injections of low doses of ChABC could not affect metastases formation. Since treatment with high concentrations of ChABC promoted metastases formation, we next 


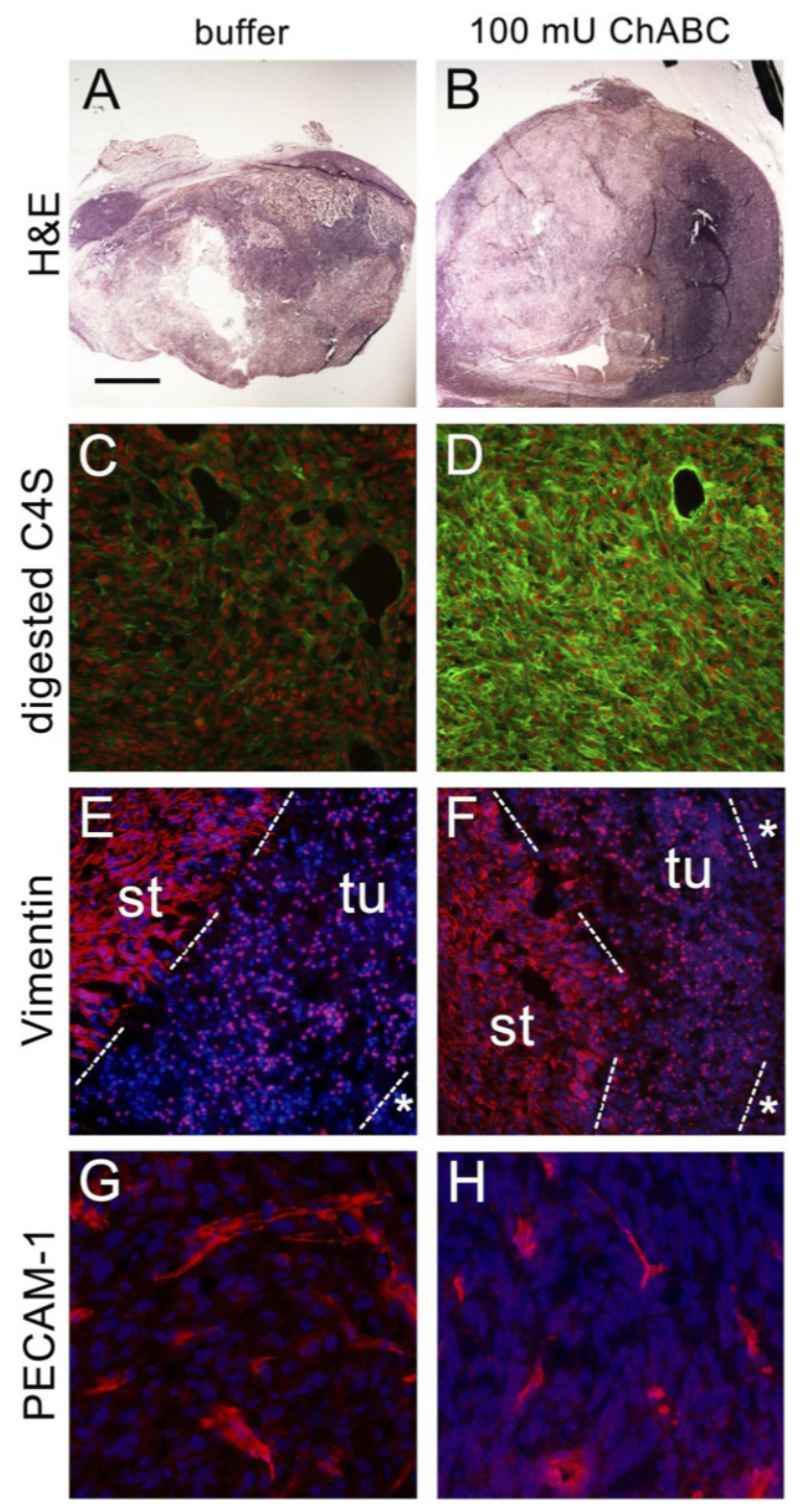

Figure 4. Histological analysis of primary tumors. Comparison of tumors treated with buffer or $100 \mathrm{mU}$ ChABC. A,B. H\&E staining. C,D. Immunofluorescence detection of digested C4S (green) showed strong staining in a

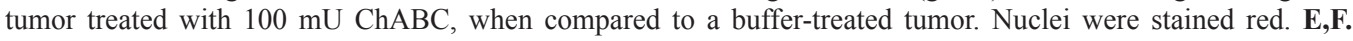
Immunofluorescence detection of vimentin (red). Both buffer- and $100 \mathrm{mU}$ ChABC-treated tumors showed strong vimentin expression in stromal compartments (st), and punctate staining in adjacent tumor compartments (tu). Tumor cells at a further distance from the stromal compartment did not express vimentin (*). Nuclei are stained blue. G,H. Immunofluorescence detection of PECAM-1 (red) to visualize tumor microvasculature. Both bufferand $100 \mathrm{mU}$ ChABC-treated tumors showed similar patterns of PECAM-1 expression. Nuclei are stained blue. Scale bars: A,B $=1 \mathrm{~mm} ; \mathrm{E}, \mathrm{F}=20 \mathrm{~mm} ; \mathrm{G}, \mathrm{H}=10 \mathrm{~mm}$. 
wanted to investigate whether loss of chondroitin sulfate could upregulate markers of invasiveness. For this, we performed immunofluorescence staining with an antibody against Vimentin, a protein induced during epithelial-to-mesenchymal transition and in tumor cells with invasive characteristics. In both buffer-injected (Figure 4E) and ChABC-injected tumors (Figure 4F), Vimentin was strongly expressed in stromal compartments (st). We also observed Vimentin staining in a more punctate pattern in breast cancer cells in the tumor compartment (tu) adjacent to the stromal compartment; however, breast cancer cells at a further distance from the stromal compartment lost Vimentin expression $(*)$. We did not observe a significant difference in Vimentin expression patterns, or levels of Vimentin expression, in buffer- versus ChABCinjected tumors. We next examined the tumor microvasculature using an antibody against platelet/endothelial cell adhesion molecule-1 (PECAM-1). Our analysis did not reveal any major differences in the pattern of the tumor microvasculature, or levels of PECAM-1 expression, in buffer-treated (Figure 4G) versus ChABC-treated (Figure 4H) tumors. These results suggested that digestion of tumor-associated chondroitin sulfates did not significantly alter the patterns of expression of invasive markers, or markers of the tumor microvasculature.

\section{DISCUSSION}

The tumor microenvironment has been suggested to be an attractive therapeutic target for treatment of cancer (Hendrix et al., 2003, 2007; Wegrowski and Maquart, 2004; Kenny et al., 2007; LaBarge et al., 2007). Chondroitin sulfate as part of the tumor microenvironment accumulates in the stromal compartment of many solid tumors, including breast tumors (Olsen et al., 1988; Kovalszky et al., 1990; Alini and Losa, 1991; Ricciardelli et al., 1997, 1999, 2009; Vijayagopal et al., 1998; Suwiwat et al., 2004; Pothacharoen et al., 2006; Sakko et al., 2008; Teng et al., 2008; Kalathas et al., 2009), the functional relevance of which had not been analyzed so far. Here, we utilized intra-tumor injections of ChABC in an orthotopic mouse model to demonstrate that enzymatic elimination of endogenous breast cancer-associated chondroitin sulfate leads to an increased metastatic burden. These results demonstrate for the first time a functional relevance of chondroitin sulfate in primary breast cancer tissue. Our data suggest that chondroitin sulfate in the primary tumor can interfere with the metastatic spreading of breast cancer cells. We observed the occurrence of large secondary tumors, and increased numbers of lung metastases in animals treated with high doses of ChABC. Markers of invasiveness and tumor microvasculature were expressed in the same pattern in primary

tumors independent of presence or absence of chondroitin sulfate, suggesting that removal of chondroitin sulfate did not increase metastases through enhanced expression of pro-metastatic proteins or major alterations in the tumor microvasculature.

Tumor-associated stroma plays a crucial role in the promotion of tumor progression through the establishment of a permissive and supportive environment (Kenny et al., 2007). Since chondroitin sulfate accumulates in the stromal compartment, an inhibitory role of chondroitin sulfate in tumor progression and metastasis was surprising. However, there are examples where molecules in the tumor stroma play dual, or even exclusively inhibitory roles in tumor progression. For example, ECM-Integrin interactions can both promote or inhibit tumor angiogenesis (Mueller and Fusenig, 2004). The ECM molecule TSP1, expressed in the tumor stroma, functions as an inhibitor of tumor angiogenesis (Mueller and Fusenig, 2004). Matrix Metalloproteinases (MMPs) can proteolytically cleave ECM molecules to generate 
peptide fragments, including angiostatin and endostatin, which inhibit tumor angiogenesis and progression. On the other hand, MMPs have also been shown to have potent pro-tumorigenic functions (Mueller and Fusenig, 2004). Thus, the impairment of breast cancer metastases by endogenous chondroitin sulfate serves as a further illustration of the complex roles of ECM molecules during tumor progression.

It is of interest to note that removal of cell surface chondroitin sulfate from mouse breast cancer, lung cancer, and osteosarcoma cells lead to decreased organ colonization of these cells after tail vein injection into mice (Li et al., 2008; Basappa et al., 2009; Cooney et al., 2011). Recent work has also shown that chondroitin sulfate on breast cancer cells play a crucial role in the interaction with Selectin proteins on endothelial cells (Kawashima et al., 2000, 2002; Luo et al., 2001; Monzavi-Karbassi et al., 2007; Cooney et al., 2011). Combined, this evidence suggests that cell surface chondroitin sulfate might play a metastasis-promoting function in the tethering of cancer cells to endothelial cells, and therefore in one aspect of cancer cell extravasation from the blood stream to target organs during the metastatic process. Combined with the results presented here, chondroitin sulfate appears to have temporally opposing functions during breast cancer progression: an anti-metastatic function in primary tumor tissue, and a pro-metastatic role during extravasation at later stages of tumor progression. Intra-tumor injections of ChABC interfered with the anti-metastatic role of chondroitin sulfate. However, once cells leave the primary tumor site, they would not be exposed to ChABC treatment anymore, and thus would be able to re-establish cell surface chondroitin sulfate moieties, which subsequently could promote extravasation steps.

Interestingly, treatment with $\mathrm{ChABC}$ in vivo has been used extensively in experimental animal models of spinal chord injury, where elimination of chondroitin sulfates by injection of $\mathrm{ChABC}$ into the lesion site promotes axonal migration and partial functional recovery (Bradbury and Carter, 2011). Thus, it appears that in both spinal cord injury and breast cancer, endogenous chondroitin sulfate can interfere with the dispersion of cells. In both situations, treatment with $\mathrm{ChABC}$ removes this block, thus promoting cell migration and dispersal.

In our experiments presented here, we observed incomplete elimination of chondroitin sulfate following our multiple $\mathrm{ChABC}$ injection regimen. In future experiments, we will aim to improve $\mathrm{ChABC}$ delivery to primary tumors. In the field of spinal cord injury, ChABCbased gene therapy approaches have recently been developed. Transgenic expression of a ChABC cDNA in glial cells near the lesion site (Cafferty et al., 2007), or expression of a closely related ChAC cDNA in bone marrow-derived mononuclear cells injected into the spinal cord lesion (Coulson-Thomas et al., 2008), lead to digestion of chondroitin sulfates, and could promote axonal regeneration. We have recently developed an epitope-tagged ChABC expression system (Klüppel, 2011), and are currently implementing the inducible transgenic expression of this expression cassette to allow spatially- and temporally-controlled expression of $\mathrm{ChABC}$, in order to identify the roles of endogenous chondroitin sulfate in tumors, embryonic development, and tissue homeostasis. In the context of solid tumors, this will enable expression of ChABC in all tumor and/or stromal cells, and thus would allow a more uniform distribution of $\mathrm{ChABC}$ than we observed with the injection regimen. Moreover, this approach will assure the continuous delivery of $\mathrm{ChABC}$ into the cellular microenvironment, while the epitope tag will enable us to follow ChABC expression.

The vast majority of cancer-related deaths are a result of the dispersion of cancer cells from the primary tumor site, and the subsequent formation of metastases in distant organs 
(Hogan et al., 2010; Kim and Baek, 2010; Mego et al., 2010). The data presented here might be suggestive of a therapeutic benefit of treatment with exogenous chondroitin sulfate to increase chondroitin sulfate levels in tumors, which might interfere with the dispersal of cancer cells and metastasis formation. From a clinical point-of-view, chondroitin sulfate is registered as a oral drug on the European market for the treatment of osteoarthritis symptoms, has been extensively studied and shown to be safe, well tolerated, and effective (Uebelhart, 2008). Future studies analyzing the incidence of cancer-related mortality in patients on a chondroitin sulfate regimen might shed light on the potential clinical benefit of chondroitin sulfate intake in breast and other cancers.

\section{ACKNOWLEDGMENTS}

The authors wish to thank Dr. Jeffrey L. Wrana, Toronto, Canada, for helpful discussions and support in the initial stages of the project. M. Klüppel wishes to acknowledge financial support from the Children's Memorial Research Center.

\section{REFERENCES}

Alini M and Losa GA (1991). Partial characterization of proteoglycans isolated from neoplastic and nonneoplastic human breast tissues. Cancer Res. 51: 1443-1447.

Basappa MS, Sugahara KN, Lee CM, ten Dam GB, et al. (2009). Involvement of chondroitin sulfate E in the liver tumor focal formation of murine osteosarcoma cells. Glycobiology 19: 735-742.

Bradbury EJ and Carter LM (2011). Manipulating the glial scar: chondroitinase ABC as a therapy for spinal cord injury. Brain Res. Bull. 84: 306-316.

Cafferty WBJ, Yang SH, Duffy PJ, Li S, et al. (2007). Functional axonal regeneration through astrocytic scar genetically modified to digest chondroitin sulfate proteoglycans. J. Neurosci. 27: 2176-2185.

Cooney CA, Jousheghany F, Yao-Borengasser A, Phanavanh B, et al. (2011). Chondroitin sulfates play a major role in breast cancer metastasis: a role for CSPG4 and CHST11 gene expression in forming surface P-selectin ligands in aggressive breast cancer cells. Breast Cancer Res. 13: R58.

Coulson-Thomas YM, Coulson-Thomas VJ, Filippo TR, Mortara RA, et al. (2008). Adult bone marrow-derived mononuclear cells expressing chondroitinase AC transplanted into CNS injury sites promote local brain chondroitin sulphate degradation. J. Neurosci. Methods 171: 19-29.

Habuchi O (2000). Diversity and functions of glycosaminoglycan sulfotransferases. Biochim. Biophys. Acta 1474: 115-127.

Heinegard D (2009). Proteoglycans and more - from molecules to biology. Int. J. Exp. Pathol. 90: 575-586.

Hendrix MJ, Seftor EA, Kirschmann DA, Quaranta V, et al. (2003). Remodeling of the microenvironment by aggressive melanoma tumor cells. Ann N. Y. Acad. Sci. 995: 151-161.

Hendrix MJ, Seftor EA, Seftor REB, Kasemeier-Kulesa J, et al. (2007). Reprogramming metastatic tumour cells with embryonic microenvironments. Nat. Rev. Cancer 7: 246-255.

Hochberg MC (2010). Structure-modifying effects of chondroitin sulfate in knee osteoarthritis: an updated meta-analysis of randomized placebo-controlled trials of 2-year duration. Osteoarthritis Cartilage 18 (Suppl 1): S28-S31.

Hogan BV, Peter MB, Shenoy H, Horgan K, et al. (2010). Intramammary lymph node metastasis predicts poorer survival in breast cancer patients. Surg. Oncol. 19: 11-16.

Iida J, Wilhelmson KL, Ng J, Lee P, et al. (2007). Cell surface chondroitin sulfate glycosaminoglycan in melanoma: role in the activation of pro-MMP-2 (pro-gelatinase A). Biochem. J. 403: 553-563.

Kalathas D, Theocharis DA, Bounias D, Kyriakopoulou D, et al. (2009). Alterations of glycosaminoglycan disaccharide content and composition in colorectal cancer: structural and expressional studies. Oncol. Rep. 22: 369-375.

Kawashima H, Hirose M, Hirose J, Nagakubo D, et al. (2000). Binding of a large chondroitin sulfate/dermatan sulfate proteoglycan, versican, to L-selectin, P-selectin, and CD44. J. Biol. Chem. 275: 35448-35456.

Kawashima H, Atarashi K, Hirose M, Hirose J, et al. (2002). Oversulfated chondroitin/dermatan sulfates containing GlcAbeta1/IdoAalpha1-3GalNAc(4,6-O-disulfate) interact with L- and P-selectin and chemokines. J. Biol. Chem. 277: 12921-12930.

Kenny PA, Lee GY and Bissell MJ (2007). Targeting the tumor microenvironment. Front Biosci. 12: 3468-3474.

Genetics and Molecular Research 10 (4): 3901-3913 (2011)

CFUNPEC-RP www.funpecrp.com.br 
Kim IS and Baek SH (2010). Mouse models for breast cancer metastasis. Biochem. Biophys. Res. Commun. 394: 443-447. Klüppel M (2010). The roles of chondroitin-4-sulfotransferase-1 in development and disease. Prog. Mol. Biol. Transl. Sci. 93: 113-132.

Klüppel M (2011). Efficient secretion of biologically active Chondroitinase ABC from mammalian cells in the absence of an N-terminal signal peptide. Mol. Cell Biochem. 351: 1-11.

Klüppel M, Vallis KA and Wrana JL (2002). A high-throughput induction gene trap approach defines C4ST as a target of BMP signaling. Mech. Dev. 118: 77-89.

Klüppel M, Wight TN, Chan C, Hinek A, et al. (2005). Maintenance of chondroitin sulfation balance by chondroitin4-sulfotransferase 1 is required for chondrocyte development and growth factor signaling during cartilage morphogenesis. Development 132: 3989-4003.

Kovalszky I, Pogany G, Molnar G, Jeney A, et al. (1990). Altered glycosaminoglycan composition in reactive and neoplastic human liver. Biochem. Biophys. Res. Commun. 167: 883-890.

Krasnokutsky S, Samuels J and Abramson SB (2007). Osteoarthritis in 2007. Bull. NYU Hosp. Jt. Dis. 65: 222-228.

Kusche-Gullberg M and Kjellen L (2003). Sulfotransferases in glycosaminoglycan biosynthesis. Curr. Opin. Struct. Biol. 13: 605-611.

LaBarge MA, Petersen OW and Bissell MJ (2007). Of microenvironments and mammary stem cells. Stem. Cell Rev. 3: 137-146.

Li F, Ten Dam GB, Murugan S, Yamada S, et al. (2008). Involvement of highly sulfated chondroitin sulfate in the metastasis of the Lewis lung carcinoma cells. J. Biol. Chem. 283: 34294-34304.

Luo J, Kato M, Wang H, Bernfield M, et al. (2001). Heparan sulfate and chondroitin sulfate proteoglycans inhibit E-selectin binding to endothelial cells. J. Cell Biochem. 80: 522-531.

Mego M, Mani SA and Cristofanilli M (2010). Molecular mechanisms of metastasis in breast cancer - clinical applications. Nat. Rev. Clin. Oncol. 7: 693-701.

Monzavi-Karbassi B, Stanley JS, Hennings L, Jousheghany F, et al. (2007). Chondroitin sulfate glycosaminoglycans as major P-selectin ligands on metastatic breast cancer cell lines. Int. J. Cancer 120: 1179-1191.

Mueller MM and Fusenig NE (2004). Friends or foes - bipolar effects of the tumour stroma in cancer. Nat. Rev. Cancer 4: 839-849.

Olsen EB, Trier K, Eldov K and Ammitzboll T (1988). Glycosaminoglycans in human breast cancer. Acta Obstet. Gynecol. Scand. 67: 539-542.

Pothacharoen P, Siriaunkgul S, Ong-Chai S, Supabandhu J, et al. (2006). Raised serum chondroitin sulfate epitope level in ovarian epithelial cancer. J. Biochem. 140: 517-524.

Ricciardelli C, Mayne K, Sykes PJ, Raymond WA, et al. (1997). Elevated stromal chondroitin sulfate glycosaminoglycan predicts progression in early-stage prostate cancer. Clin. Cancer Res. 3: 983-992.

Ricciardelli C, Quinn DI, Raymond WA, McCaul K, et al. (1999). Elevated levels of peritumoral chondroitin sulfate are predictive of poor prognosis in patients treated by radical prostatectomy for early-stage prostate cancer. Cancer Res. 59: 2324-2328.

Ricciardelli C, Sakko AJ, Stahl J, Tilley WD, et al. (2009). Prostatic chondroitin sulfate is increased in patients with metastatic disease but does not predict survival outcome. Prostate 69: 761-769.

Sakko AJ, Butler MS, Byers S, Reinboth BJ, et al. (2008). Immunohistochemical level of unsulfated chondroitin disaccharides in the cancer stroma is an independent predictor of prostate cancer relapse. Cancer Epidemiol. Biomark. Prev. 17: 2488-2497.

Suwiwat S, Ricciardelli C, Tammi R, Tammi M, et al. (2004). Expression of extracellular matrix components versican, chondroitin sulfate, tenascin, and hyaluronan, and their association with disease outcome in node-negative breast cancer. Clin. Cancer Res. 10: 2491-2498.

Teng YH, Tan PH, Chia SJ, Zam NA, et al. (2008). Increased expression of non-sulfated chondroitin correlates with adverse clinicopathological parameters in prostate cancer. Mod. Pathol. 21: 893-901.

Thiele H, Sakano M, Kitagawa H, Sugahara K, et al. (2004). Loss of chondroitin 6-O-sulfotransferase-1 function results in severe human chondrodysplasia with progressive spinal involvement. Proc. Natl. Acad. Sci. U. S. A. 101: 10155-10160.

Uebelhart D (2008). Clinical review of chondroitin sulfate in osteoarthritis. Osteoarthritis Cartilage 16 (Suppl 3): S19-S21.

Vijayagopal P, Figueroa JE and Levine EA (1998). Altered composition and increased endothelial cell proliferative activity of proteoglycans isolated from breast carcinoma. J. Surg. Oncol. 68: 250-254.

Viloria-Petit AM and Wrana JL (2010). The TGFbeta-Par6 polarity pathway: linking the Par complex to EMT and breast cancer progression. Cell Cycle 9: 623-624.

Viloria-Petit AM, David L, Jia JY, Erdemir T, et al. (2009). A role for the TGFbeta-Par6 polarity pathway in breast cancer progression. Proc. Natl. Acad. Sci. U. S. A. 106: 14028-14033.

Wandel S, Juni P, Tendal B, Nuesch E, et al. (2010). Effects of glucosamine, chondroitin, or placebo in patients with 
osteoarthritis of hip or knee: network meta-analysis. BMJ 341: c4675.

Wegrowski Y and Maquart FX (2004). Involvement of stromal proteoglycans in tumour progression. Crit. Rev. Oncol. Hematol. 49: 259-268. 